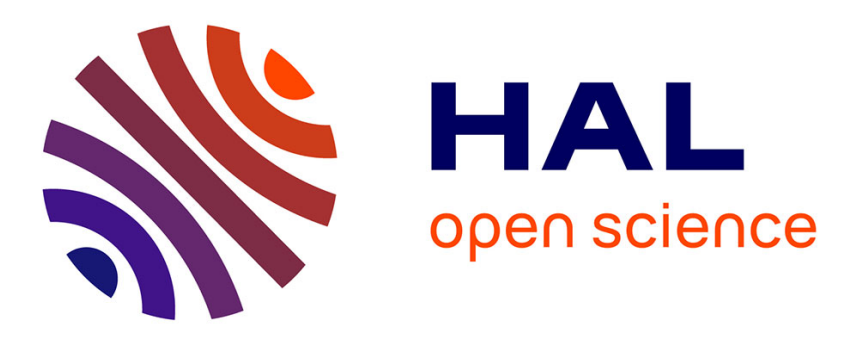

\title{
CupCarbon: A New Platform for the Design, Simulation and 2D/3D Visualization of Radio Propagation and Interferences in IoT Networks
}

Ahcène Bounceur, Laurent Clavier, Pierre Combeau, Olivier Marc Marc, Rodolphe Vauzelle, Arnaud Masserann, Julien Soler, Reinhardt Euler, Taha Ahmed Mohammed Al Wajeeh, Vyas Devendra, et al.

\section{To cite this version:}

Ahcène Bounceur, Laurent Clavier, Pierre Combeau, Olivier Marc Marc, Rodolphe Vauzelle, et al.. CupCarbon: A New Platform for the Design, Simulation and 2D/3D Visualization of Radio Propagation and Interferences in IoT Networks. IEEE Consumer Communications \& Networking Conference, Jan 2018, Las Vegas, United States. hal-01704408

\author{
HAL Id: hal-01704408 \\ https://hal.science/hal-01704408
}

Submitted on 8 Feb 2018

HAL is a multi-disciplinary open access archive for the deposit and dissemination of scientific research documents, whether they are published or not. The documents may come from teaching and research institutions in France or abroad, or from public or private research centers.
L'archive ouverte pluridisciplinaire HAL, est destinée au dépôt et à la diffusion de documents scientifiques de niveau recherche, publiés ou non, émanant des établissements d'enseignement et de recherche français ou étrangers, des laboratoires publics ou privés. 


\title{
CupCarbon: A New Platform for the Design, Simulation and 2D/3D Visualization of Radio Propagation and Interferences in IoT Networks
}

\author{
Ahcène Bounceur ${ }^{1}$, Laurent Clavier ${ }^{2}$, Pierre Combeau ${ }^{3}$, Olivier Marc ${ }^{4}$, Rodolphe Vauzelle ${ }^{3}$ \\ Arnaud Masserann ${ }^{4}$, Julien Soler ${ }^{4}$, Reinhardt Euler ${ }^{1}$, Taha Alwajeeh ${ }^{1,3}$, Vyas Devendra ${ }^{3}$, Umber Noreen ${ }^{1,2}$ \\ Emilie Soret ${ }^{2}$, Massinissa Lounis ${ }^{1}$ \\ ${ }^{1}$ Lab-STICC CNRS UMR 6285 - Université de Bretagne Occidentale, Brest, France \\ ${ }^{2}$ IEMN, IRCICA USR 3380, Lille, France \\ ${ }^{3}$ Xlim CNRS UMR 7252 - Université de Poitiers, France \\ ${ }^{4}$ Virtualys, Brest, France
}

\begin{abstract}
The number of connected devices is growing and in the near future it is expected to become extremely large in cities. As a consequence, using simulators to study and prepare a project of installing new networks before their real deployment is of great importance. They can help to predict some important information like signal overload or the feasibility of the deployment in terms of location, interferences, communication and cost. In this paper we present a new architecture for the platform CupCarbon, developed within the research project PERSEPTEUR. The main objective of this platform is to design and simulate Wireless Sensor Networks dedicated to Smart-city and IoT applications. It allows to validate distributed algorithms in a 2D/3D environment, taking account of the city buildings in which to deploy the network, the mobiles, and using accurate models of radio propagation and interferences in that environment.

Index Terms-Wireless Sensor Networks, IoT, Simulation, Design, Propagation, Interferences, Distributed algorithms.
\end{abstract}

\section{INTRODUCTION}

A wireless sensor node is mainly composed of a microcontroller, a number of sensors, a battery and a radio module. This later is designed to be low energy consuming since it is the part that consumes the most. One way to reduce this consumption is to reduce the radio communication range, as is the case of the ZigBee 802.15.4 standard, which is designed especially for Wireless Sensor Network (WSN) applications. Since the radio range is small, it is required to use many sensor nodes in order to route any information to the coordinator or to the base station. Since these nodes communicate wirelessly, it is suggested to use a low cost communication algorithm. Many protocols have been presented in the literature, like Leach, many to one, AODV, etc.

Another way is to route messages directly to the base station in only one hop. This is possible by using low cost and long distance wireless communication modules as is the case with the LoRa and SigFox protocols.

Since it is expected that in the near future, more than $50 \%$ of the population will live in cities, the number of connected devices will increase significantly. This will lead to the use of a huge number of devices communicating wirelessly. Therefore, our environment will be saturated in terms of communication signals and spectrum. This can be harmful for our health as well as for any future WSN installation. That is why it is necessary to thoroughly study any new project before its real installation. To do this, simulators must be used to study the impact, in terms of wireless signal overload and security, of any new installation before its real deployment.

The existing simulators, like NS-2/3 [1], OMNET (Castalia) [2], TOSSIM [3], OPNET [4], WSNet [5], etc., are used mainly to develop new routing protocols. However, in the context of smart-cities and IoT, their radio channel and interference models are very simple and do not take into account the real city environment. Besides, they do not integrate the visualization in order to validate easily the developed algorithm. The main contributions of this multidisciplinary work is to keep simulation times very short while taking into account the 3D, an accurately simulated radio channel with the impact of the obstacles on the environment and a realistic evaluation of the interference. The platform has been developed with the following objectives:

- Study the deployment of wireless sensor networks by taking into account the mobility and the availability of the spectrum,

- Simulate performances and services of a wireless sensor network in a 2D/3D realistic environment,

- Study the feasibility of communication, the reliability of the network and its cost,

- Detect any interference zones in order to improve the quality of the deployment,

- Simulate accurately and quickly the radio propagation in a real urban environment,

- Visualize the simulation result in order to debug and validate a developed algorithm.

The remainder of the paper is organized as follows: Section II presents the proposed platform. In Section III the architecture and the simulator CupCarbon is presented and finally, Section IV concludes the paper. 


\section{THE PRESENTATION OF THE PLATFORM}

As shown in Fig. 1, the proposed platform is composed of 4 main parts: a 2D/3D environment block, an interference block, a radio channel block and an implementation block.

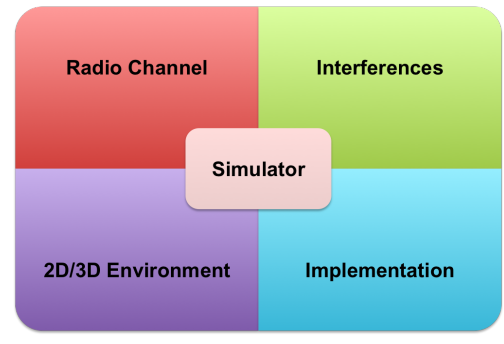

Fig. 1. Main parts of the CupCarbon platform.

These parts are detailed in the following sections.

\section{A. Radio channel block}

CupCarbon integrates two radio propagation models. The first one is a $2.5 \mathrm{D}$ based on a point to zone acceleration structure called visibility tree [11], [12] (cf. Fig. 2). It allows to estimate very quickly the channel attenuation and the channel Impulse Response (IR) according to a large number of receivers. The second one is a full $3 \mathrm{D}$ ray-tracing associated with a Monte-Carlo algorithm (cf. Fig. 3). It is able to exploit the slight diffuse behavior of the reflection surfaces but not the diffraction yet, and it is more time consuming. A specific implementation on GPU is being realized and should allow a significant reduction of the computation time.

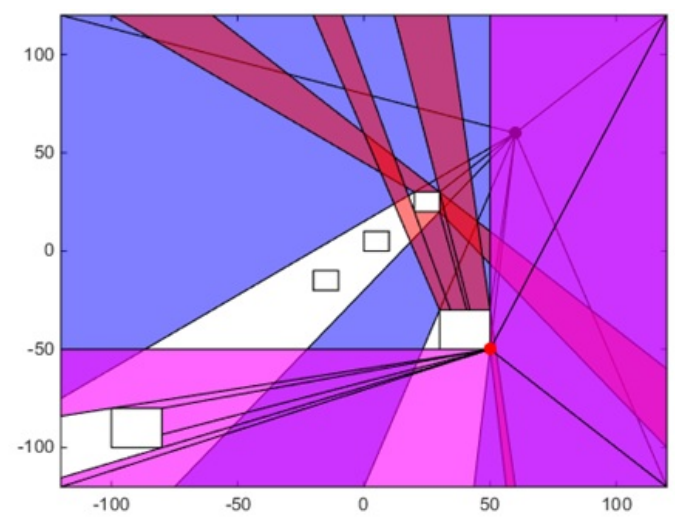

Fig. 2. Tree visibility concept: visibility zones (purple), reflection zones (red), and diffraction zones (magenta).

\section{B. Interference block}

1) PHY Layer: A significant originality in CupCarbon is to be able to implement the true PHYsical layer of the communication. Baseband models for three different standards have been integrated: the ZigBee [8], WiFi [9] and LoRa [10] protocols. The evaluation of a link quality can now be based on accurate transmission conditions that take into account the

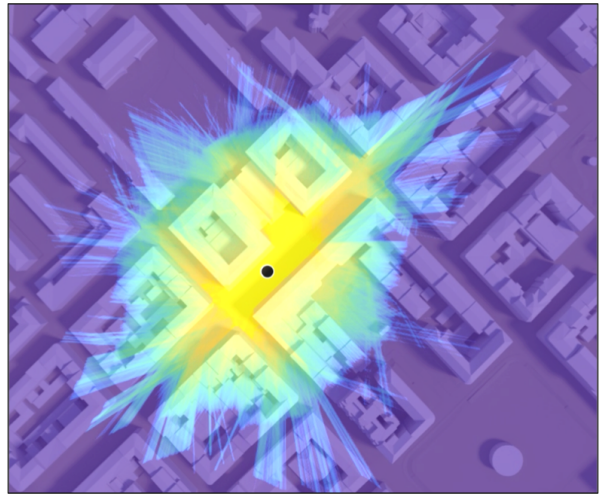

Fig. 3. Top view of a 3D ray tracing based radio propagation.

radio channel and the data encoding. This approach however can be very time consuming if we need to generate all the signals in the city. That is why we propose in the next section some statistical approaches to model interference.

2) Interference: Interference is a significantly limiting factor in dense networks. In Fig. 4, a ZigBee packet is transmitted in an environment with Bluetooth and WiFi sources of interference. The short Bluetooth signal corrupts the ZigBee packets which need to be re-transmitted. An exact evaluation of this interference is feasible and CupCarbon allows a generation of all the received signals. However, such an approach can become very time consuming due to a possibly high number of interfering sources: signals have to be generated, channels to be simulated and the addition of all the received contributions creates the interference. To avoid too heavy calculations, it is possible to randomly generate the interference. However, as shown in many papers (see [6], [7] for instance) a Gaussian model is not accurate. One solution is to accurately evaluate the main contributions of the interference and to randomly generate the global contribution of the less impacting interferers. Another possibility is to generate the global interference with a single distribution. Our proposal is to use $\alpha$-stable distributions with parameters depending on the radio channel statistics and the interferer density. Future work will include a study of spatial dependence in the interference model using Copulae [15].

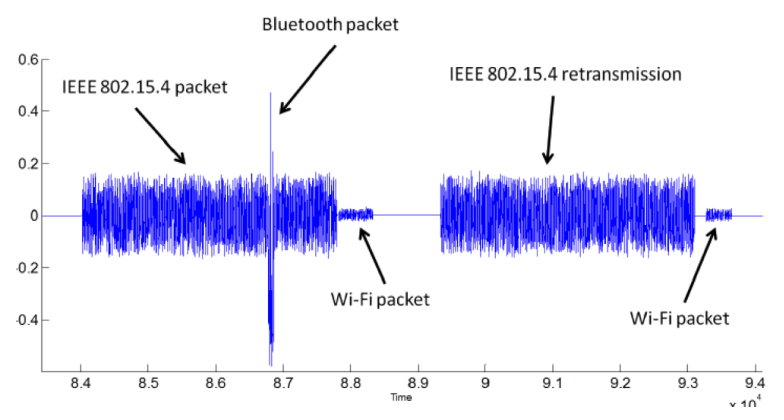

Fig. 4. Interfered signal. 


\section{2D/3D Environment block}

The 2D and 3D visualization is very important for the deployment of the different nodes of the network. The 3D environment helps to achieve an accurate deployment in which the elevation can be taken into account. This elevation generates different radio propagation and interferences. The $2 \mathrm{D}$ environment is useful for simulation debugging and validation. Fig. 5 shows an example of a city displayed in the 3D environment of the CupCarbon simulator.

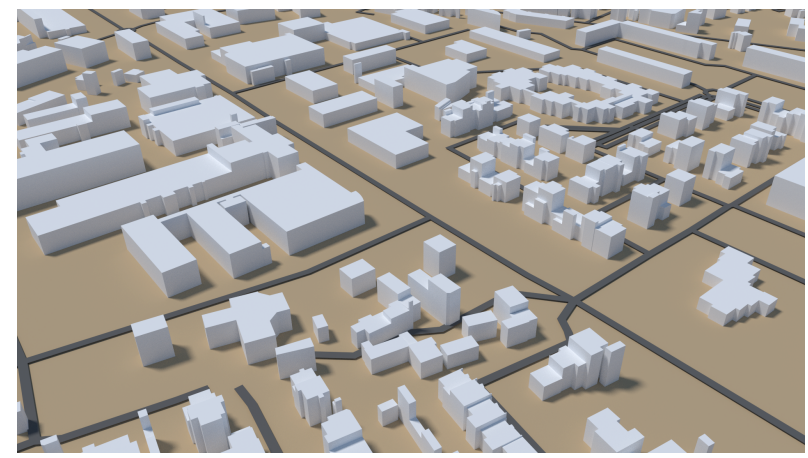

Fig. 5. Example of a 3D part of a city as displayed in CupCarbon.

The 3D environment of CupCarbon is composed of ground elevation, buildings and various objects like sensor nodes. The ground elevation model can be imported into a CubCarbon project using heightmaps, which are simple grayscale images and meta data, providing GPS coordinates and bounds (min and max altitude) of the heightmaps. The ground elevation can also be obtained using external web services such as Google Elevation API. Once the elevation is imported, a bilinear interpolation is performed to compute a triangle mesh. Top shapes of buildings can be imported from the Overpass API provided by OSM. Then these shapes will be extruded on the ground elevation. Sensor nodes are simply represented by spheres of various colors and sizes, depending on their respective states and types. Links between sensors are shown by lines. Ground, buildings and sensor node meshes are then sent to the graphic card using the OpenGL API. Since CupCarbon is programmed in Java, we have used the JOGL (Java OpenGL) library in order to create and use the OpenGL context.

\section{Implementation block}

The platform was designed with a modular structure to simplify replacement and customization of specific parts of the simulator. The modular structure provides two very important abilities. The first one is the simplification and the customization of the target architecture. It is easy to modify parts of the architecture, while keeping the rest unchanged. This makes the platform useful for simulating specific wireless sensor networks. The second ability is to promote multiple implementations of a given module, which allows users to switch rapidly and easily between different versions.

\section{THE PROPOSED ARCHITECTURE}

The architecture in Fig. 6 shows the main modules of the CupCarbon simulator. They are described as follows:

- 2D/3D City model module: this module represents a digital format of a city. It contains the different informations about the buildings, roads, places, etc. It is the main part of the simulator that is first presented to the user. It allows to deploy the different sensor nodes of the network and it is used to calculate the interferences and the signal propagations.

- Mobility module: It allows to create the routes of the mobiles. A mobile can be just a device without any communicating system or it can be a sensor node or a device with a sensor node. The mobility can be fixed in advance, where the mobile follows a given trajectory. It can be determined in the script according to a given situation (e.g., detection of a target, an abnormal sensed value, etc.) which allows to perform intelligent mobility.

- Network module: This module allows to design the wireless sensor network which will be simulated.

- Communication Script module: This module represents the interpreter of the SenScript language used to program each node of the network. The simulator will execute the instructions of the script of each node.

- Radio Channel Propagation module: This module is used to calculate the channel attenuation and its impulse response according to each couple of nodes in the network. These data allow to determine for each couple of sensor nodes whether the level of interference or the transmitted packet is received or not. Therefore, it allows to determine whether they can communicate in the format of a matrix (cf. Propagation And Connection Matrix block).

- Interference module: This module is used to determine for each sent message whether it will be received or not by the receiver. It uses the models presented above. This is where the PHY layer is implemented.

- Simulation module: This module is the kernel of the proposed architecture. It is based on discrete event simulation. The events are generated either by executing each instruction of the script of each sensor node or by a real event like mobility or a natural event like temperature, gas, etc.

An existing version of the simulator CupCarbon is available online [13], [14]. Fig. 7 shows its main graphical user interface. It is ergonomic and designed to be used in an easy and intuitive way.

\section{CONCLUSION}

We have presented a new architecture of a platform called CupCarbon for the simulation of Wireless Sensor Networks dedicated to Smart-cities and Internet of Things. The originality of this platform is the visualization of the simulation results during the execution process, which takes into account the radio propagation and the interferences generated by the sensor nodes deployed on a 3D digital city. The integrated 


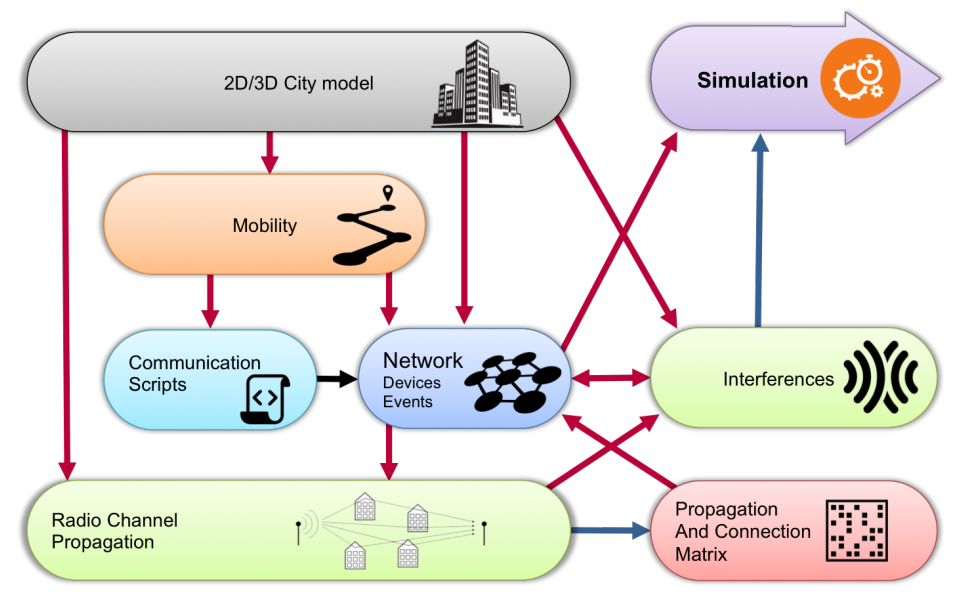

Fig. 6. Main parts of the architecture of the CupCarbon platform.

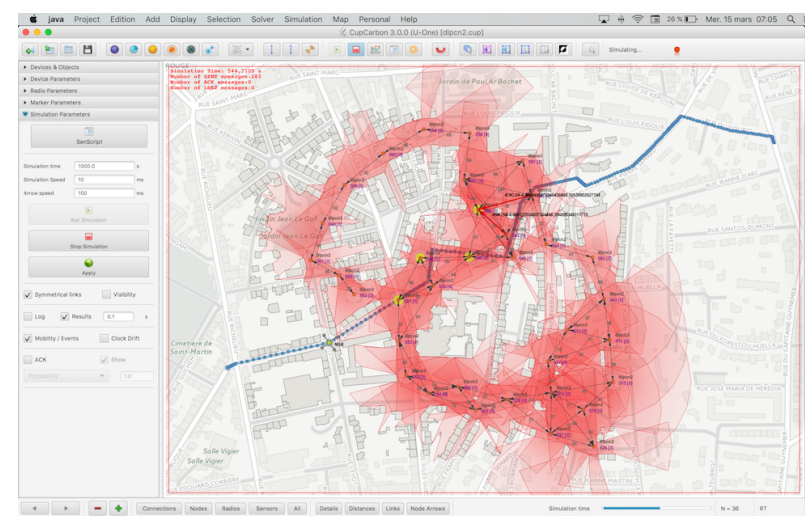

Fig. 7. Graphical user interface of the CupCarbon platform.

radio models are developed within the framework of this work. They are accurate and fast to simulate. We are working on a new version where the virtual nodes will be replaced by real ones, like Arduino and Raspberry, and where the simulation will be executed on these nodes. The existing CupCarbon version will serve for their programming and the visualization. The main advantages of this platform is the possibility to prepare quickly any real IoT network without programming each real sensor node individually, and to allow an accurate study of some parameters on the basis of real networks instead of virtual ones.

\section{ACKNOWLEDGMENT}

This project is supported by the French National Research Agency ANR PERSEPTEUR - REF: ANR-14-CE24-0017.

\section{REFERENCES}

[1] The Network Simulator, NS-2 [Online]. Available: http://www.isi.edu/nsnam/n

[2] OMNET++ discrete event simulator. [Online]. Available: http://www.omnetpp.org
[3] P. Levis, N. Lee, M. Welsg, D. Culler, TOSSIM: Accurate and Scalable Simulation of Entire TinyOS Applications. In Proc. 1st ACM Int. Conf. Embedded Networked Sensor Systems (SenSys), Los Angeles, CA, pp. 126-137, 2003.

[4] F. Desbrandes, S. Bertolotti, and L. Dunand. OPNET 2.4: An environment for communication network modeling and simulation, In Proceedings of the European Simulation Symposium, pp. 609-614, Delft, Nertherlands, October 1993.

[5] WSNet Overview. http://wsnet.gforge.inria.fr/overview.html. accessed June 2012.

[6] M. L. de Freitas, M. Egan, L. Clavier, A. Goupil, G. W. Peters and N. Azzaoui, "Capacity Bounds for Additive Symmetric alpha-Stable Noise Channels," in IEEE Transactions on Information Theory, vol. 63, no. 8, pp. 5115-5123, Aug. 2017.

[7] H. E. Ghannudi, L. Clavier, N. Azzaoui, F. Septier and P. a. Rolland, $" \alpha$-stable interference modeling and cauchy receiver for an IR-UWB Ad Hoc network," in IEEE Transactions on Communications, vol. 58, no. 6 , pp. 1748-1757, June 2010.

[8] Umber Noreen, Ahcne Bounceur, Laurent Clavier, Rahim Kacimi. Performance Evaluation of IEEE 802.15.4 PHY with Impulsive Network Interference in CupCarbon Simulator. IEEE International Symposium on Networks, Computers and Communications (ISNCC 2016), May 2016, Tunis, Tunisia.

[9] Umber Noreen, Ahcne Bounceur, Laurent Clavier. Integration of OFDM Based Communication System with Alpha-Stable Interference using CupCarbon Simulator. International Conference on Internet of Things and Cloud Computing, Mar 2016, Cambridge, United Kingdom. ICC, 2016, International Conference on Internet of Things and Cloud Computing.

[10] Umber Noreen, Ahcne Bounceur, Laurent Clavier. A Study of LoRa Low Power and Wide Area Network Technology. 3rd IEEE International Conference on Advanced Technologies for Signal and Image Processing (ATSIP'2017), May 2017, Fez, Morocco.

[11] Alwajeeh Taha, Pierre Combeau, Ahcne Bounceur, Rodolphe Vauzelle. Efficient Method for Associating Radio Propagation Models with Spatial Partitioning for Smart City Applications. International Conference on Internet of Things and Cloud Computing, Mar 2016, Cambridge, United Kingdom.

[12] Taha Alwajeeh, Pierre Combeau, Rodolphe Vauzelle and Ahcène Bounceur, A High-Speed 2.5D Ray-Tracing Propagation Model for Microcellular Systems, Application: Smart Cities, In the 11th IEEE European Conference on Antennas and Propagation (EuCAP 2017), 1924 March 2017, Paris, France.

[13] Mehdi, K., Lounis, M., Bounceur, A., and Kechadi, T. CupCarbon: A Multi-Agent and Discrete Event Wireless Sensor Network Design and Simulation Tool, In IEEE 7th International Conference on Simulation Tools and Techniques (SIMUTools'14), Lisbon, Portugal, 2014.

[14] CupCarbon simulator, http://www.cupcarbon.com

[15] Emilie Soret, Laurent Clavier, Gareth W. Peters, Ido Nevat, Francois Septier, "SIMO communication with impulsive and dependent interference - the Copula receiver.", GRETSI 2017. 